\title{
Rapid, efficient, and economical synthesis of PET tracers in a droplet microreactor: application to O-(2-[ $\left.{ }^{18} \mathrm{~F}\right]$ fluoroethyl)-L- tyrosine $\left(\left[{ }^{18} \mathrm{~F}\right] \mathrm{FET}\right)$
}

Ksenia Lisova ${ }^{1,2,3} \mathbb{D}$, Bao Ying Chen ${ }^{1,2,3}$, Jia Wang ${ }^{2,3,4}$, Kelly Mun-Ming Fong ${ }^{2,3}$, Peter M. Clark ${ }^{1,2,3}$ and R. Michael van Dam ${ }^{1,2,3,4^{*}}$

\author{
* Correspondence: mvandam@ \\ mednet.ucla.edu \\ 'Physics in Biology and Medicine \\ Interdepartmental Graduate \\ Program, University of California, \\ Los Angeles, Los Angeles, CA, USA \\ ${ }^{2}$ Crump Institute for Molecular \\ Imaging, University of California, Los \\ Angeles, Los Angeles, CA, USA \\ Full list of author information is \\ available at the end of the article
}

\begin{abstract}
Background: Conventional scale production of small batches of PET tracers (e.g. for preclinical imaging) is an inefficient use of resources. Using O- $\left(2-\left[{ }^{18} \mathrm{~F}\right]\right.$ fluoroethyl)-Ltyrosine $\left(\left[{ }^{18} \mathrm{~F}\right] \mathrm{FET}\right)$, we demonstrate that simple microvolume radiosynthesis techniques can improve the efficiency of production by consuming tiny amounts of precursor, and maintaining high molar activity of the tracers even with low starting activity.

Procedures: The synthesis was carried out in microvolume droplets manipulated on a disposable patterned silicon "chip" affixed to a heater. A droplet of $\left[{ }^{18} \mathrm{~F}\right]$ fluoride containing $\mathrm{TBAHCO}_{3}$ was first deposited onto a chip and dried at $100^{\circ} \mathrm{C}$. Subsequently, a droplet containing $60 \mathrm{nmol}$ of precursor was added to the chip and the fluorination reaction was performed at $90^{\circ} \mathrm{C}$ for $5 \mathrm{~min}$. Removal of protecting groups was accomplished with a droplet of $\mathrm{HCl}$ heated at $90^{\circ} \mathrm{C}$ for $3 \mathrm{~min}$. Finally, the crude product was collected in a methanol-water mixture, purified via analytical-scale radio-HPLC and formulated in saline. As a demonstration, using $\left[{ }^{18} \mathrm{~F}\right] \mathrm{FET}$ produced on the chip, we prepared aliquots with different molar activities to explore the impact on preclinical PET imaging of tumor-bearing mice.

Results: The microdroplet synthesis exhibited an overall decay-corrected radiochemical yield of $55 \pm 7 \%(n=4)$ after purification and formulation. When automated, the synthesis could be completed in 35 min. Starting with $<370$ MBq of activity, $\sim 150$ $\mathrm{MBq}$ of $\left[{ }^{18} \mathrm{~F}\right] \mathrm{FET}$ could be produced, sufficient for multiple in vivo experiments, with high molar activities (48-119 GBq/ $/$ mol). The demonstration imaging study revealed the uptake of $\left[{ }^{18} \mathrm{~F}\right] \mathrm{FET}$ in subcutaneous tumors, but no significant differences in tumor uptake as a result of molar activity differences (ranging $0.37-48 \mathrm{GBq} / \mu \mathrm{mol}$ ) were observed.

Conclusions: A microdroplet synthesis of $\left[{ }^{18} \mathrm{~F}\right] \mathrm{FET}$ was developed demonstrating low reagent consumption, high yield, and high molar activity. The approach can be expanded to tracers other than $\left.{ }^{[8} \mathrm{F}\right] \mathrm{FET}$, and adapted to produce higher quantities of the tracer sufficient for clinical PET imaging.

Keywords: Radiochemistry, Microfluidics, FET, Amino acid imaging, Droplet synthesis, Molar activity, Pre-clinical imaging, Automation, Droplet microreactor
\end{abstract}




\section{Background}

Positron emission tomography (PET) is a non-invasive molecular imaging tool based on the use of positron-emitting isotopes to track the position and dynamics of biologically relevant molecules in the body. PET provides high-sensitivity quantitative visualization of physiological parameters in vivo, such as metabolic rate, receptor density, gene expression, or blood flow, which makes it a versatile and potent tool for clinical diagnosis, treatment planning, treatment monitoring, as well as research (Aboagye et al. 2001; Ambrosini et al. 2009; Ciernik et al. 2003; Kitson et al. 2009; Phelps 2000).

Safe preparation of various target-specific PET tracers is a complex and expensive process, requiring skilled personnel operating expensive automated radiosynthesis equipment within radiation-shielded "hot cells". With conventional apparatus, in which the chemistry is carried out in $\mathrm{mL}$ volume scales, relatively high reagent amounts $(1 \mathrm{~s}$ to $10 \mathrm{~s}$ of $\mathrm{mg}$ ) are needed to achieve a sufficient concentration for good reaction yield in a short time, and for $\left[{ }^{18} \mathrm{~F}\right]$ fluoride chemistry high amounts of radioactivity (10s of GBq) are needed to achieve high molar activity (Sergeev et al. 2018a). These factors contribute to inefficient use of resources in the preparation of small batches of tracers, such as needed for preclinical imaging, or for a single clinical PET scan, where much of the prepared batch would be wasted.

On the other hand, emerging microfluidic radiosynthesis methods require much lower amounts of reagents and radionuclide, and through substantially reduced instrument size and cost, have the potential to significantly reduce costs and resources needed for radiopharmaceutical production. Microscale reactions also tend to be faster and, due to the low precursor mass used, the crude products can be purified with simpler methods (e.g. analytical-scale high-performance liquid chromatography (HPLC) or cartridge instead of semi-preparative HPLC). These advantages are especially relevant for smaller batch production of PET tracers, but can also benefit the production of larger batches (Chao et al. 2018b).

Of the several different microfluidic approaches reported in the last decade (Elizarov 2009; Keng and van Dam 2015; Miller et al. 2010; Pascali and Matesic 2016; Rensch et al. 2013), microvolume reaction approaches offer the greatest potential for reagent and instrument reductions (Dooraghi et al. 2013; Elizarov et al. 2010; Iwata et al. 2018; Keng et al. 2016; Lebedev et al. 2012; Wang et al. 2017). A particular configuration we are exploring is performing reactions in microliter-sized droplets on simple Teflon-coated silicon microfluidic chips, which has advantages of simple operation, low-cost disposable chips, and a compact system size, which reduces the necessary shielding. Previous work has shown application of this method for the rapid and efficient synthesis of $\left[{ }^{18}\right.$ F]FDG and $\left[{ }^{18} \mathrm{~F}\right]$ Fallypride (Wang et al. 2017). In this paper, we demonstrate further versatility of this approach by adapting the macroscale synthesis of O-(2-[ $\left.{ }^{18} \mathrm{~F}\right]$ fluoroethyl)-L-tyrosine $\left(\left[{ }^{18} \mathrm{~F}\right] \mathrm{FET}\right)$ to this platform, and then use the produced $\left[{ }^{18} \mathrm{~F}\right] \mathrm{FET}$ for pre-clinical imaging.

$\left[{ }^{18} \mathrm{~F}\right] \mathrm{FET}$ is an amino acid PET probe (Wester et al. 1999), finding use in glioma imaging (Langen et al. 2017) as well as providing a route for protein labeling with fluorine-18 (Yanai et al. 2019). The radiosynthesis of $\left[{ }^{18} \mathrm{~F}\right] \mathrm{FET}$ from the commercially available precursor (2S)-O-(2'-tosyloxyethyl)- $\mathrm{N}$-trityl-tyrosine-tert-butyl ester (TET) consists of a radiofluorination step followed by a hydrolysis step. The conventional synthesis typically results in good radiochemical yields (RCYs), ranging from 19 to $64 \%$ (Bourdier et al. 2011; Bouvet et al. 2012; Hamacher and Coenen 2002; Iwata et al. 2018; 
Lakshminarayanan et al. 2016). Some efforts have been made to carry out the synthesis in microfluidic format. Bouvet et al. performed the reaction in a commercial flow radiochemistry system using either microwave or heat activation of the reaction. An RCY of $50 \%$ was obtained with only $59 \mathrm{nmol}$ of precursor in a $30 \mu \mathrm{L}$ reaction in $<45 \mathrm{~min}$ (Bouvet et al. 2012), but to scale to larger production amounts (e.g., $>200 \mathrm{MBq}$ ) would require longer synthesis times and higher precursor amounts. Iwata et al. performed batch synthesis in $10-20 \mu \mathrm{L}$ volumes $(180-350 \mathrm{nmol}$ of precursor) within a small glass vial by first loading a larger volume of methanolic solution containing $\left[{ }^{18} \mathrm{~F}\right]$ fluoride and phase transfer catalyst, evaporating the solvent, then adding the small volume of precursor solution and performing the reaction (Iwata et al. 2018). Yields of up to $64 \pm 11 \%(n=3 \sim 6)$ were reported at scales of $<400 \mathrm{MBq}$. An automated procedure for this method was not described and may be challenging in practice due to the difficulty of manipulating small volumes in what is essentially a conventional apparatus.

We report a simple and rapid method for $\left[{ }^{18} \mathrm{~F}\right] \mathrm{FET}$ synthesis based on microvolume droplet approach. The probe production with this method results in high RCY and high molar activity using a very small amount of precursor and low starting activity. The low precursor amount enables purification via analytical, rather than semi-preparative, scale HPLC. This low-cost approach allowed us to carry out a large dynamic imaging study of up to 8 mice within a single day, thus demonstrating that the method will be a favorable option for pre-clinical studies of $\left[{ }^{18} \mathrm{~F}\right] \mathrm{FET}$.

\section{Materials and methods}

\section{Materials}

\section{Reagents and supplies}

For the radiochemistry portion of this work, no-carrier-added $\left[{ }^{18} \mathrm{~F}\right]$ fluoride was produced by the ${ }^{18} \mathrm{O}(\mathrm{p}, \mathrm{n}){ }^{18} \mathrm{~F}$ reaction from $\left[{ }^{18} \mathrm{O}\right] \mathrm{H}_{2} \mathrm{O}$ (84\% isotopic purity, Zevacor Pharma, Noblesville, IN, USA) in an RDS-112 cyclotron (Siemens; Knoxville, TN, USA) at $11 \mathrm{MeV}$ using a $1 \mathrm{~mL}$ tantalum target with havar foil. Acetonitrile (MeCN; anhydrous, 99.8\%), methanol ( $\mathrm{MeOH}$; anhydrous, 99.8\%), ethanol (EtOH; 200 proof, > 99.5\%), hydrochloric acid $(\mathrm{HCl} ; 1 \mathrm{M})$, thexyl alcohol (2,3-dimethyl-2-butanol, 98\%), trifluoroacetic acid (TFA, 99\%), deionized (DI) water, phosphate buffered saline (PBS; pH 7.4) were purchased from Millipore Sigma (St. Louis, MO, USA). Saline (0.9\% sodium chloride injection, USP) was obtained from Hospira Inc. (Lake Forest, IL, USA). All reagents were used as received without further purification. $18 \mathrm{M} \Omega$ water was obtained from a purification system (RODI-C-12BL, Aqua solutions, Inc., Georgia, USA). Tetrabutylammonium bicarbonate $0.075 \mathrm{M}$ ( $\left.\mathrm{TBAHCO}_{3},>99 \%\right)$, (2S)-O-(2'-tosyloxyethyl)N-trityl-tyrosine-tert-butyl ester (TET, > 95\%) precursor, O-2-fluoroethyl-L-tyrosine standard (FET-HCl, > 95\%) were purchased from ABX GmbH (Radeberg, Germany).

To perform uptake assays, GS025 and GBM39 cells were kindly provided by Dr. David Nathanson (UCLA), the ParcB3 cells were provided by Dr. Peter Clark (UCLA), and the HCT-15 and HCC827 cells were purchased from ATCC (Manassas, VA, USA). Poly-L-lysine, protease inhibitor $\left(\mathrm{cOmplete}^{\mathrm{Tx}}\right)$, Hank's balanced salt solution (HBSS; $10 \times$ ), and fetal bovine serum (FBS),were purchased from Millipore Sigma (St. Louis, MO, USA). 96 well plates, 96 well filter plates, $0.25 \%$ trypsin, $100 \times$ penicillinstreptomycin $\left(10,000 \mathrm{U} / \mathrm{mL}\right.$, Gibco $\left.{ }^{\mathrm{mm}}\right)$, RPMI-1640 medium $\left(1 \times\right.$, Gibco $\left.^{\mathrm{Tm}}\right)$, GlutaMAX ${ }^{\mathrm{mm}}-$ 
I (100×, Life Technologies), Dulbecco's Modified Eagle Medium (DMEM/F12), (100×), epidermal growth factor recombinant human protein (EGF), fibroblast growth factor recombinant human protein (FGF-Basic), heparin, and B27 supplement (50x) were purchased from Thermo Fisher (Waltham, MA, USA).

\section{Analytical methods}

A calibrated ion chamber (CRC 25-PET, Capintec, Florham Park, NJ, USA) was used to perform radioactivity measurements. For radio-thin-layer chromatography (TLC) analysis, TLC plates (Baker-flex silica gel IB-F sheets $2.5 \times 7.5 \mathrm{~cm}$, J.T. Baker, Phillipsburg, $\mathrm{NJ})$ were spotted with $1 \mu \mathrm{L}$ samples of the crude intermediate, crude final product, or purified final product, and were developed in $80 \% \mathrm{v} / \mathrm{v} \mathrm{MeCN}$ in $\mathrm{H}_{2} \mathrm{O}$, and then scanned with a radio-TLC scanner (miniGita star, Raytest, Inc., Wilmington, NC, USA), or with a Cerenkov luminescence imaging system (Dooraghi et al. 2013). Retention factors of the observed radioactive species were: $0.0\left(\left[{ }^{18} \mathrm{~F}\right]\right.$ fluoride), $0.3\left(\left[{ }^{18} \mathrm{~F}\right] \mathrm{FET}\right)$, and 0.8 (fluorinated intermediate).

Radio-HPLC analysis and purification was performed on an analytical-scale Smartline HPLC system (Knauer, Berlin, Germany) with $200 \mu \mathrm{L}$ injection loop, a pump (Model 1000), degasser (Model 5050), a UV detector (Model 2500) and a radiometric detector (Bioscan B-FC-4000, Bioscan Inc., Washington DC, USA). Samples were separated using a C18 column (Luna, $4.6 \times 250 \mathrm{~mm}, 5 \mu \mathrm{m}$, Phenomenex, Torrance, CA, USA) with guard column (SecurityGuard C18, Phenomenex) at a flow rate of $1 \mathrm{~mL} / \mathrm{min}$. UV absorbance was measured at $269 \mathrm{~nm}$. Using $10 \% \mathrm{v} / \mathrm{v}$ EtOH in $18 \mathrm{M} \Omega \mathrm{H}_{2} \mathrm{O}$ mobile phase, the expected retention time of $\left[{ }^{18} \mathrm{~F}\right]$ fluoride was between 2 and $3 \mathrm{~min}$, and around $5 \mathrm{~min}$ for $\left[{ }^{18} \mathrm{~F}\right] \mathrm{FET}$. The fluorinated intermediate was eluted off the column using $100 \% \mathrm{MeCN}$.

\section{Microfluidic systems}

Radiochemistry was performed in droplet format on the surface of microfluidic chips comprising a silicon wafer with a patterned Teflon AF coating. The detailed fabrication was previously reported (Wang et al. 2017). A combination of hydrophobic (Teflon) and hydrophilic (exposed silicon wafer) regions allows liquid droplets to be manipulated or maintained in a desired location to perform reactions.

One type of chip, used for the synthesis optimization studies, had a $4 \mathrm{~mm}$ circular hydrophilic region (i.e. Teflon coating etched away) serving as a reaction site (Fig. 1a). During use, the chips were mounted to a temperature control platform comprising a ceramic heater affixed to a Peltier device, which was in turn mounted on a heat sink with a fan. A thin layer of thermal paste was present between all device components to ensure good thermal contact. Reagents were loaded and product was collected manually via pipette with 10 or $200 \mu \mathrm{L}$ tips.

Another type of chip, used for automated synthesis, had six radial tapered hydrophilic pathways leading toward a central hydrophilic reaction site (Fig. 1b). The chip was similarly mounted to a temperature-control platform, but reagents were added via electronically controlled piezoelectric actuators around the periphery of the chip and crude product was collected by a retractable needle. The tapered pathways spontaneously 


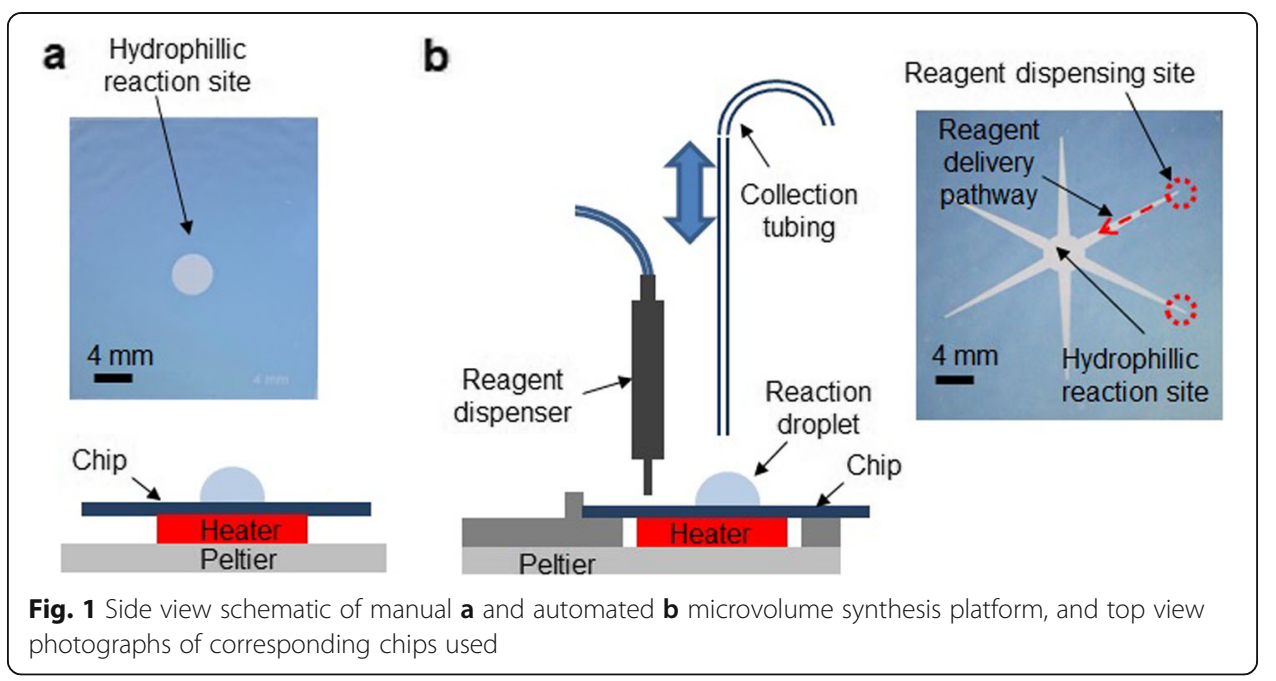

transport reagent droplets from the periphery to the center of the chip. Complete details of this setup were reported previously (Wang et al. 2017).

\section{Methods}

\section{Microscale radiosynthesis and purification of $\left[{ }^{18} \mathrm{~F}\right] \mathrm{FET}$}

The microscale synthesis was adapted from previously described macroscale protocols (Bourdier et al. 2011; Hamacher and Coenen 2002) by scaling down reagent volumes (Fig. 2). Cyclotron produced $\left[{ }^{18} \mathrm{~F}\right]$ fluoride $(37-740 \mathrm{MBq}$ in $\sim 10-20 \mu \mathrm{L})$ was mixed with $110 \mathrm{nmol}$ of $\mathrm{TBAHCO}_{3}$ (i.e., $1.5 \mu \mathrm{L}$ of $75 \mathrm{mM}$ solution), deposited on the chip and then evaporated to dryness at $100{ }^{\circ} \mathrm{C}$. After cooling to $30^{\circ} \mathrm{C}, 10 \mu \mathrm{L}$ of $6 \mathrm{mM}$ TET $(60 \mathrm{nmol})$ in $1: 1 \mathrm{v} / \mathrm{v}$ MeCN:thexyl alcohol was added to the chip. The reaction mixture was heated at $90{ }^{\circ} \mathrm{C}$ for $5 \mathrm{~min}$, and then cooled to $30^{\circ} \mathrm{C}$. Next, $20 \mu \mathrm{L}$ of $1 \mathrm{M} \mathrm{HCl}$ was added and the deprotection reaction was performed by heating to $90^{\circ} \mathrm{C}$ for $3 \mathrm{~min}$. The crude product was recovered by adding $20 \mu \mathrm{L}$ of $1: 1 \mathrm{v} / \mathrm{v} \mathrm{MeOH}: \mathrm{H}_{2} \mathrm{O}$ and collecting from the chip. The collection process was repeated a total of 4 times to ensure high recovery of the crude product. After synthesis, the product was diluted to $150-175 \mu \mathrm{L}$ using HPLC mobile phase $(10 \% \mathrm{v} / \mathrm{v}$ EtOH in $18 \mathrm{M} \Omega$ water) and purified via analytical-scale radio-HPLC. The product peak was collected (typically $1.0-1.5 \mathrm{~min}$ duration) into a sterile glass vial. Solvent was evaporated by heating the vial to $120^{\circ} \mathrm{C}$ with an oil bath and applying a

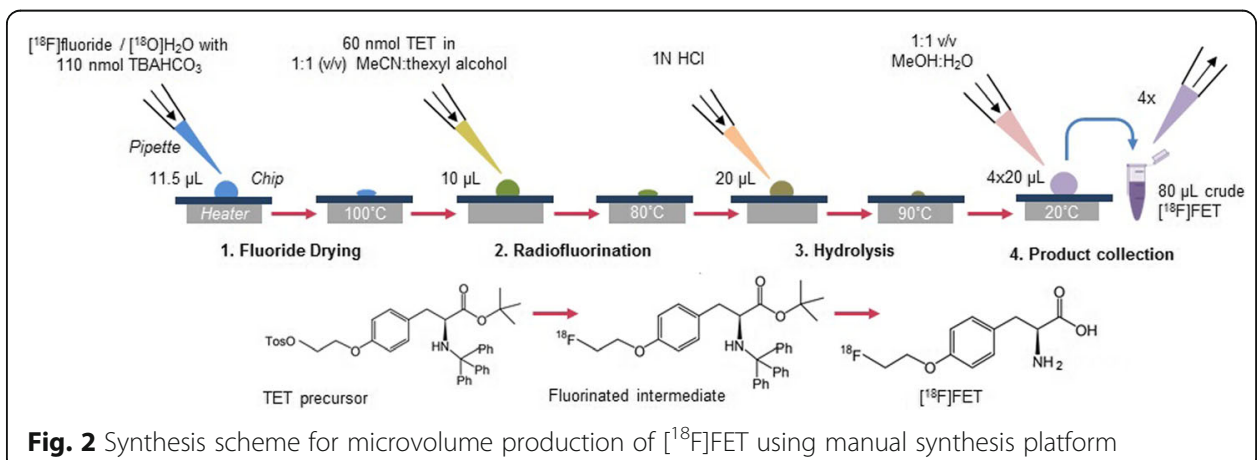

Fig. 2 Synthesis scheme for microvolume production of $\left[{ }^{18} \mathrm{~F}\right] \mathrm{FET}$ using manual synthesis platform 
nitrogen stream above the surface of the solvent. When dry (typically after 10-15 min), the $\left[{ }^{18} \mathrm{~F}\right] \mathrm{FET}$ was then resuspended either in sterile saline for in vivo imaging, or $\mathrm{pH} 7.4$ phosphate-buffered saline (PBS) for cell uptake experiments. Numerous intermediate measurements were taken during synthesis to carefully analyze its performance (see details in Electronic supplementary material (ESM), Additional file 1: Sects. 1-2).

\section{In vitro probe uptake}

In vitro uptake of $\left[{ }^{18} \mathrm{~F}\right] \mathrm{FET}$ was compared across two glioblastoma cell lines (GS025, GBM39), a prostate cancer cell line (ParcB3), a lung cancer cell line (HCC827), and a colon cancer cell line (HCT-15). The GS025, GBM39, and ParcB3 suspension cells were grown in stem cell media, and the HCC827 and HCT-15 adherent cells were grown in supplemented RPMI. The suspension cells were plated into 96-well plates and adherent cells into 96 -well filter plates at 150,000 cells $/ \mathrm{mL}$ concentration in $1 \mathrm{x}$ HBSS. $\left[{ }^{18} \mathrm{~F}\right] \mathrm{FET}$ was diluted to a concentration of $370 \mathrm{~Bq} / \mu \mathrm{L}$ with either PBS (for uptake experiments) or PBS containing $5 \mathrm{mM}$ FET (for blocking experiments). Cell uptake experiments were performed by adding $100 \mu \mathrm{L}\left[{ }^{18} \mathrm{~F}\right] \mathrm{FET}(37 \mathrm{kBq})$ to each of a set of cell wells $(n=4)$, and blocking experiments (to confirm specificity) were performed by adding $100 \mu \mathrm{L}\left[{ }^{18} \mathrm{~F}\right] \mathrm{FET}(37 \mathrm{kBq})$ with FET $(500 \mathrm{nmol})$ to each of a set of cell wells $(n=4)$. The cells were incubated at $37^{\circ} \mathrm{C}$ for $10 \mathrm{~min}$, then transferred into individual gamma counter tubes and sample radioactivity was measured on a gamma counter (WIZARD 3" 1480, Perkin Elmer, Waltham, MA, USA). The uptake values were normalized to total protein amounts for each sample (complete details of the procedure are provided in the ESM, Additional file 1: Sect. 3). The statistical significance of the values was validated by a two-tailed $\mathrm{T}$ test $(p<0.05)$.

\section{In vivo preclinical imaging}

Male NOD scid gamma (NSG) mice $\sim 7$ week-old were obtained from the UCLA Department of Radiation Oncology. These mice $(n=10)$ were engrafted with $0.5 \times 10^{6}$ HCC827 cells suspended in a 1:1 (v/v) mixture of supplemented RPMI media and Corning $^{\bullet}$ Matrigel $^{\circ}$ Basement Membrane Matrix (Corning Life Sciences) in the left and right shoulders.

To perform dynamic PET imaging, mice were kept under $2 \%$ isoflurane anesthesia during the tracer uptake for $60 \mathrm{~min}$. Mice were injected with 1.5-3.1 MBq of the tracer, and were scanned 4 at a time using the recently developed HiPET scanner (Gu et al. 2017). The first study was performed with 4 mice injected with probe of different molar activities in a range of $1.5-36 \mathrm{GBq} / \mu \mathrm{mol}(n=1$ each). The second study with 8 mice covered molar activities ranging from $0.4-48 \mathrm{GBq} / \mu \mathrm{mol}(n=2 \mathrm{each}$ ) (see Additional file 1: ESM, Sect. 4 for details). The concentration of FET in blood was estimated to range between 0.02 and $3.5 \mu \mathrm{M}$ assuming $2 \mathrm{~mL}$ average mouse blood volume. All mice received 10 min CT scans (CrumpCAT (Taschereau et al. 2014)) following the PET imaging experiment. After PET/CT image registration, regions of interest (ROI) were drawn with AMIDE version 1.0.5 software, and the results were analyzed by comparing mean intensity values of the tumors and other regions across different time points (12 frames of 5 min each) (details on image analysis are described in ESM, Additional file 1: Sect. 5). 


\section{Results}

\section{Microscale $\left[{ }^{18} \mathrm{~F}\right] \mathrm{FET}$ synthesis optimization and automation}

To adapt the 2-step synthesis of $\left[{ }^{18} \mathrm{~F}\right] \mathrm{FET}$ from the macroscale to the microscale, the precursor and base quantities were initially scaled down nearly 300-490-fold from values reported in conventional synthesis (Bourdier et al. 2011; Hamacher and Coenen 2002), i.e. to $75 \mathrm{nmol}$ of $\mathrm{TBAHCO}_{3}(1 \mu \mathrm{L}, 0.075 \mathrm{M})$ and $30 \mathrm{nmol}$ of the TET precursor in $20 \mu \mathrm{L}$. We used $\mathrm{TBAHCO}_{3}$ rather than $\mathrm{K}_{222} / \mathrm{K}_{2} \mathrm{CO}_{3}$ (Bourdier et al. 2011; Iwata et al. 2018) based on the suggestion by Hamacher and Coenen (2002), who observed higher yields due to the lower basicity and reduced competing elimination reaction. One significant change we made was altering the fluorination reaction solvent. The syntheses reported by Hamacher and Coenen (2002), Bourdier et al. (2011) and Lakshminarayanan et al. (2016) all used $\mathrm{MeCN}$ as the fluorination solvent, but in the droplet format, we found that the $\mathrm{MeCN}$ evaporated very quickly, resulting in poor yields. After exploring several solvent combinations that have been previously reported in droplet reactions (Keng and van Dam 2015), a 1:1 v/v mixture of MeCN and thexyl alcohol was selected. During early syntheses the fluorination temperature was set at $80^{\circ} \mathrm{C}$, slightly lower than what has been reported in conventional syntheses (i.e., $85^{\circ} \mathrm{C}$ (Hamacher and Coenen 2002) or $100^{\circ} \mathrm{C}$ (Bourdier et al. 2011)) to further mitigate evaporation, and the reaction time was set for $5 \mathrm{~min}$. Under these conditions, fluorination yield was $36 \pm$ $7 \%(n=4)$.

Investigation of the ratio of base to precursor (Fig. 3a) indicated that the originally chosen ratio $(\sim 2.5)$ was close to optimal: a steep drop in fluorination efficiency was observed for base to precursor ratios below 1.7 and higher than 2.5. When fluorinating with $110 \mathrm{nmol}$ of $\mathrm{TBAHCO}_{3}$ per $60 \mathrm{nmol}$ of precursor $\left(1.9\right.$ ratio) at $80^{\circ} \mathrm{C}$ the fluorination yield reached $50 \pm 1 \%(n=4)$. Increasing the temperature to $90{ }^{\circ} \mathrm{C}$ further improved the fluorination yield to $63 \pm 3 \%(n=4)$ (Fig. 4). Lower reaction temperature $\left(75^{\circ} \mathrm{C}\right.$ ) resulted in similar yield as the $80^{\circ} \mathrm{C}$ reaction, though solvent evaporation was slightly reduced (Fig. 4). Later, the reaction volume was reduced to $10 \mu \mathrm{L}$ keeping the same amount $(60 \mathrm{nmol})$ of precursor per reaction to make it more compatible with chip chemistry, however no significant change in reaction yield was observed.

For the deprotection step, we initially attempted to use TFA as reported by Hamacher and Coenen (2002) and Bouvet et al. (2012); however, we observed rapid
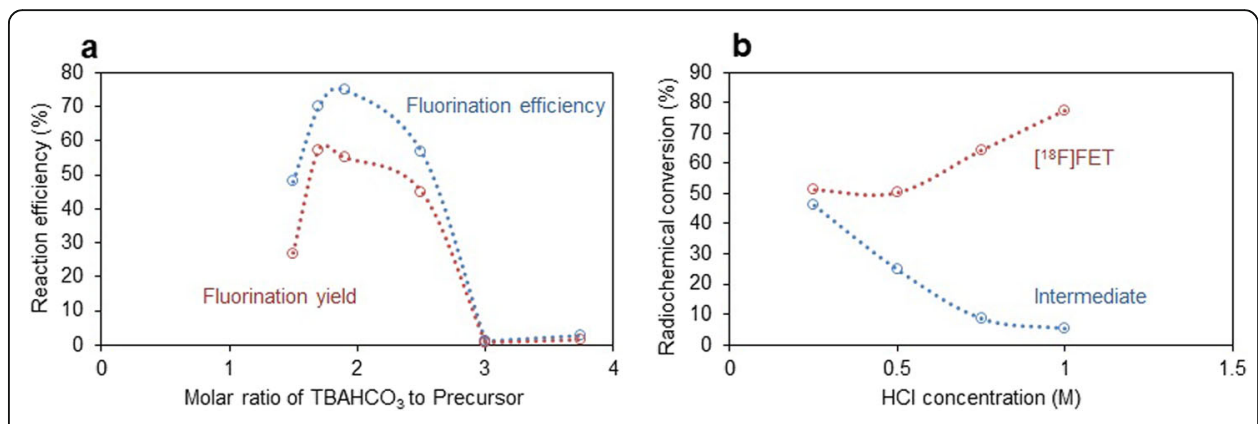

Fig. 3 a Effect of base to precursor ratio on fluorination efficiency and fluorination yield ( $n=1$ for each data point). Syntheses carried out at $80^{\circ} \mathrm{C}$ for 5 min with $30 \mathrm{nmol}$ or $60 \mathrm{nmol}$ of precursor. $\mathbf{b}$ Effect of deprotectant $(10 \mu \mathrm{L} \mathrm{HCl})$ concentration on deprotection reaction at $90^{\circ} \mathrm{C}$ for $3 \mathrm{~min}(n=1$ for each condition). Synthesis performed with $60 \mathrm{nmol}$ precursor and $110 \mathrm{nmol} \mathrm{TBAHCO}_{3}$ at $90^{\circ} \mathrm{C}$ for $5 \mathrm{~min}$ 


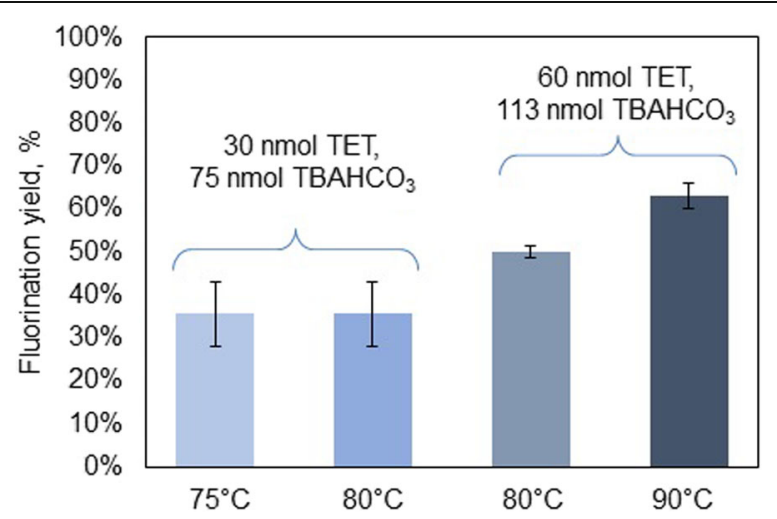

Fig. 4 Results of initial optimization of fluorination conditions. Error bars represent standard deviations $(n=4)$

evaporation of TFA and low deprotection efficiency. We then explored the use of $\mathrm{HCl}$, as reported by Bourdier et al. (2011) and Lakshminarayanan et al. (2016). Using a deprotection reagent volume of $10 \mu \mathrm{L}$ heated for $3 \mathrm{~min}$ at $90^{\circ} \mathrm{C}$, we explored the effect of different $\mathrm{HCl}$ concentrations (Fig. 3b). Higher concentrations resulted in more complete deprotection of the intermediate. The use of $10 \mu \mathrm{L}$ of $1.0 \mathrm{M} \mathrm{HCl}$ was sufficient to deprotect most of the intermediate $(\sim 94 \%)$. Increasing the volume from 10 to $20 \mu \mathrm{L}$ led to improved hydrolysis and was used in all subsequent experiments. Conveniently, the acid nearly fully evaporates during the hydrolysis step leaving only trace amounts of liquid, obviating the need for neutralization.

The manual synthesis of the crude product, under optimized conditions, required $24 \pm 2 \min (n=4)$. The collection efficiency was $64 \pm 5 \%(n=4)$ and radiochemical conversion of $\left[{ }^{18} \mathrm{~F}\right] \mathrm{FET}$ was $92 \pm 4 \%(n=4)$ resulting in crude RCY of $59 \pm 7 \%(n=4)$. Fluorination yield was estimated to be $62 \pm 8 \%(n=4)$, and hydrolysis efficiency was $96 \pm 2 \%(n=4)$. Only $1.3 \pm 0.5 \%(n=4)$ of the starting activity was attributed to residual activity on the chip after collection of the crude product, though an additional loss of $35 \pm 6 \%(n=4)$ was observed, potentially corresponding to loss of unreacted $\left[{ }^{18} \mathrm{~F}\right]$ fluoride in the form of $\left[{ }^{18} \mathrm{~F}\right] \mathrm{HF}$ during the acidic deprotection step.

Production of $\left[{ }^{18} \mathrm{~F}\right] \mathrm{FET}$ for imaging was performed using this manual protocol, followed by purification by analytical HPLC $(\sim 5 \mathrm{~min})$ and formulation $(10-15 \mathrm{~min})$, resulting in an overall synthesis time of $40 \mathrm{~min}$. The loss during purification and formulation was $7 \pm 3 \%(n=4)$ and overall decay corrected RCY was $55 \pm 7 \%(n=4)$. The identity of the purified product was confirmed via analytical radio-HPLC by coinjection with the reference standard. Radiochemical purity of the final product as determined via radio-HPLC was $>98 \%$. Molar activity was $48-119 \mathrm{GBq} / \mu \mathrm{mol}$ at the end of synthesis.

We also performed the synthesis using the automated droplet radiosynthesizer (i.e. with the passive transport microfluidic chips) and observed a crude decaycorrected RCY of $54 \pm 6 \%(n=5)$ (a detailed comparison of the performance of the manual and automated droplet synthesis processes is summarized in Table 1). In general, the performance was very similar, the main difference being slightly lower collection efficiency with the automated procedure. An advantage of the automated synthesis is that the synthesis of the crude product was completed in a shorter time (5 min less). 
Table 1 Summary of performance of microdroplet synthesis of $\left[{ }^{18} \mathrm{~F}\right] \mathrm{FET}$ with optimized manual operation or automated operation. All values are decay-corrected unless otherwise specified

\begin{tabular}{lll}
\hline & Manual $(n=4)$ & Automated $(n=5)$ \\
\hline Collection efficiency (\%) & $64 \pm 5$ & $59 \pm 10$ \\
Residual chip activity (\%) & $1.3 \pm 0.5$ & $3 \pm 1$ \\
Volatile activity loss (\%) & $35 \pm 6$ & $38 \pm 11$ \\
Fluorination yield (\%) & $62 \pm 8$ & $59 \pm 10$ \\
Radiochemical conversion to FET (\%) & $92 \pm 4$ & $93 \pm 6$ \\
Deprotection efficiency (\%) & $96 \pm 2$ & $93 \pm 6$ \\
Crude RCY (\%) & $59 \pm 7$ & $54 \pm 6$ \\
Crude synthesis time (min) & $24 \pm 2$ & $19 \pm 2$ \\
Crude RCY, non-decay-corrected (\%) & $51 \pm 6$ & $48 \pm 5$ \\
\hline
\end{tabular}

In vivo imaging at varying molar activities of $\left[{ }^{18} \mathrm{~F}\right] \mathrm{FET}$

As a demonstration of the ability to perform a preclinical imaging study with $\left[{ }^{18} \mathrm{~F}\right] \mathrm{FET}$ produced using the microscale method, we prepared $\left[{ }^{18} \mathrm{~F}\right] \mathrm{FET}$ of different molar activities to investigate the impact on in vivo imaging. It has been previously seen with imaging of $\left[{ }^{18} \mathrm{~F}\right]$ Fallypride that molar activity can significantly affect the PET imaging contrast in the striata of the brain (Sergeev et al. 2018b), whereas variations in molar activity of $\left[{ }^{18} \mathrm{~F}\right]$ FDOPA were reported not to impact the imaging of neuroendocrine tumors (Kuik et al. 2015). $\left[{ }^{18} \mathrm{~F}\right] \mathrm{FET}$ is one of the major fluorine-18 labeled amino acids used in glioma imaging, grading and therapy planning. $\left[{ }^{18} \mathrm{~F}\right] \mathrm{FET}$ is an L-tyrosine analogue, and it helps to visualize amino acid transport activity that is upregulated in many growing tumors (Langen et al. 2006, 2017).

To perform experiments, samples with different molar activities were prepared from a single batch of $\left[{ }^{18} \mathrm{~F}\right] \mathrm{FET}$. The batch was divided into four aliquots, then each aliquot was spiked with different amounts of the reference standard and saline to achieve different molar activity values with the same radioactivity concentration (details of the preparation are included in the ESM, Additional file 1: Sect. 4).

The cell uptake comparison among few different cell lines had shown that the lung cancer cell line HCC827 had a significantly higher probe uptake than any of the other cell lines tested (GS025, GBM39, ParcB3, HCT-15) (Fig. 5) and was used for in vivo study. Subcutaneous tumor HCC827 xenograft models had reached sufficient tumor size for imaging $(\sim 4 \mathrm{~mm}$ diameter) after 36 days when an initial imaging experiment was performed (ESM, Additional file 1: Fig. S3), followed by another study at 50 days post-implantation (ESM, Additional file 1: Fig. S4). Dynamic PET/CT scans were performed with injections of different molar activities. In all cases, the signal in the blood was high after injection and decreased over time. Muscle and tumor uptake rose gradually and plateaued at $\sim 30 \mathrm{~min}$, remaining nearly constant until the end of the scan. No bone uptake was observed in scans, confirming the lack of in vivo defluorination. Combined dynamic imaging data is summarized in ESM, Additional file 1: Fig. S5-7.

The tumor to blood ratio increased during the first 15-20 $\mathrm{min}$ and then remained nearly constant for the rest of the scan, while the tumor to muscle ratio remained nearly constant throughout the scan (Additional file 1: Fig. S7). Qualitatively, it is apparent there is no strong correlation between the tumor uptake ratios and the molar activity values. Tumors imaged at low molar activity were as easily visible as tumors 


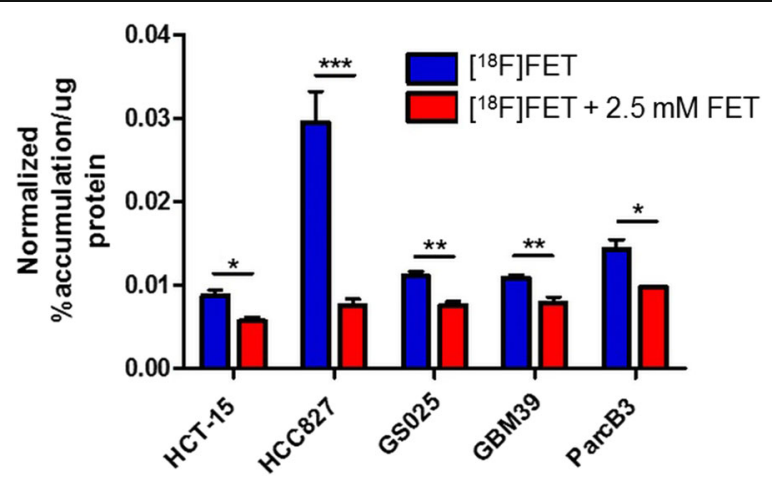

Fig. 5 Accumulation of $\left[{ }^{18} \mathrm{~F}\right] \mathrm{FET}$ in different cell lines. Error bars represent standard deviation $(n=4)$. $\left({ }^{*}\right) \mathrm{p}<$ $0.05,\left(^{* *}\right) p<0.01,\left(^{* *}\right) p<0.001$. The red bars indicate incubation with both $\left[{ }^{18} \mathrm{~F}\right] \mathrm{FET}$ and $2.5 \mathrm{mM}$ FET reference standard to establish specificity

imaged at high molar activity of the injected probe. The uptake ratios averaged during the final 30-60 min of the scans summarized for different molar activity values did not exhibit any correlation either (Fig. 6). The statistically insignificant correlation between uptake ratios and molar activity was confirmed using a Spearman correlation test $\left(\mathrm{r}_{\mathrm{s}}=\right.$ -0.3 for tumor to muscle ratio, $r_{s}=0.1$ for tumor to blood ratio).

\section{Discussion}

\section{Microscale synthesis}

The microscale synthesis described here was performed quickly, reliably and in high yield, allowing production of the tracer for pre-clinical studies. A comparison of the performance of the microvolume synthesis compared to conventional synthesis is included in Table 2 . The consumption of reagents was reduced drastically $(>150 \times$ less precursor) compared to conventional methods, while still achieving comparable RCY. Though optimization runs (requiring numerous intermediate measurements), and batches for imaging (where molar activity adjustments were needed at the end) took longer to prepare, the fully-automated microvolume synthesis can be completed in 35

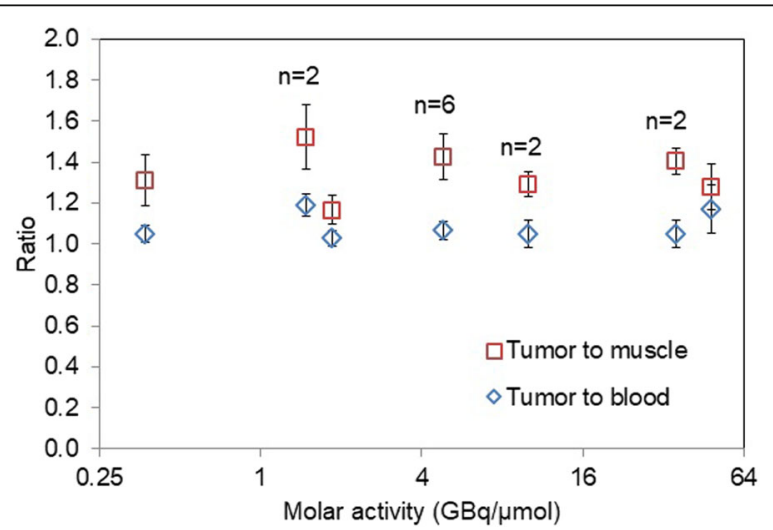

Fig. 6 Tumor to muscle and tumor to blood ratios averaged for all tumors within the same molar activity value group ( $n=4$ except as otherwise indicated) and averaged over the dynamic imaging data from 30 to $60 \mathrm{~min}$. Error bars represent standard deviation 
min (19 min synthesis +6 min purification via analytical-HPLC +10 min formulation). This is significantly faster than macroscale synthesis methods, and is a significant advantage when considering non-decay-corrected $\mathrm{RCY}$. The short synthesis time originates from the smaller reaction volume, which enables faster temperature change and shorter solvent removal times, as well as from the low precursor mass, which enables the use of analytical scale HPLC purification rather than semi-preparative. Interestingly, the droplet method also resulted in shorter synthesis time and higher yield compared to recent reports of $\left[{ }^{18} \mathrm{~F}\right] \mathrm{FET}$ synthesis in smaller volumes (10s of microliters) using manual liquid manipulation or flow-through reactors (Table 2).

Under optimized conditions, a batch of $\left[{ }^{18} \mathrm{~F}\right] \mathrm{FET}$ suitable for preclinical imaging throughout the day (e.g. 37-110 MBq; assuming 0.93-7.4 MBq per injection for 5-10 mice) could be produced on the microscale platform starting with only $110-330 \mathrm{MBq}$ of $\left[{ }^{18} \mathrm{~F}\right]$ fluoride. Limiting the activity to relatively low levels in this manner could have significant advantages for shielding the apparatus (i.e., thinner shielding would be adequate) and possibly operating the synthesis outside of a hot cell.

The droplet synthesis (even with starting activities lower than $0.74 \mathrm{GBq}$ ) resulted in high molar activities, comparable to the values achieved on macroscale synthesizers starting with $>30 \mathrm{GBq}$ of fluoride-18. It should be appreciated that, when the starting activity is scaled down in macroscale radiosynthesizers, one observes a linear decrease in the resulting molar activity (Sergeev et al. 2018a). Thus, high amounts of starting activity must often be used in macroscale synthesizers, even if only a relatively small amount of the final tracer is needed. Compared to microscale synthesis, this can result in higher cost of the radioisotope, and the need for considerably more shielding to work with the higher activity levels.

Overall, the microvolume synthesis of radiopharmaceuticals has a number of advantages over conventional scale radiosynthesizers such as more compact apparatus, reduced shielding, rapid synthesis, high yield, and efficient use of radioisotope. These advantages have the potential to drive down the costs of materials and infrastructure, which can be a significant benefit for limited resource settings or preclinical tracer production. Another advantage - low precursor consumption - not only helps to simplify the purification step, but can also represent a significant cost reduction, especially for tracers with expensive precursors, or in situations where precursor is scarce, such as the development of novel tracers or optimization of synthesis protocols. While the strengths of this technology are in reducing costs of small batches of tracers, e.g. to support in vitro or preclinical studies, various microfluidic technologies are constantly improving and expanding their applications in the radiopharmacy field, and could also lead to improvements in the efficiency of clinical PET tracer production in the future (Pascali et al. 2013), such as enabling the production of additional tracers with minimal need for extra space or capital.

\section{$\left[{ }^{18} \mathrm{~F}\right] \mathrm{FET}$ imaging}

Over the range tested $(0.37-48 \mathrm{GBq} / \mu \mathrm{mol})$, the molar activity had no statistically significant effect on imaging of subcutaneous HCC827 tumors. $\left[{ }^{18} \mathrm{~F}\right] \mathrm{FET}$ accumulates in cells following transport by $\mathrm{Na}^{+}$-dependent and -independent amino acid transporters and is not incorporated into proteins over the time course of the imaging experiments 


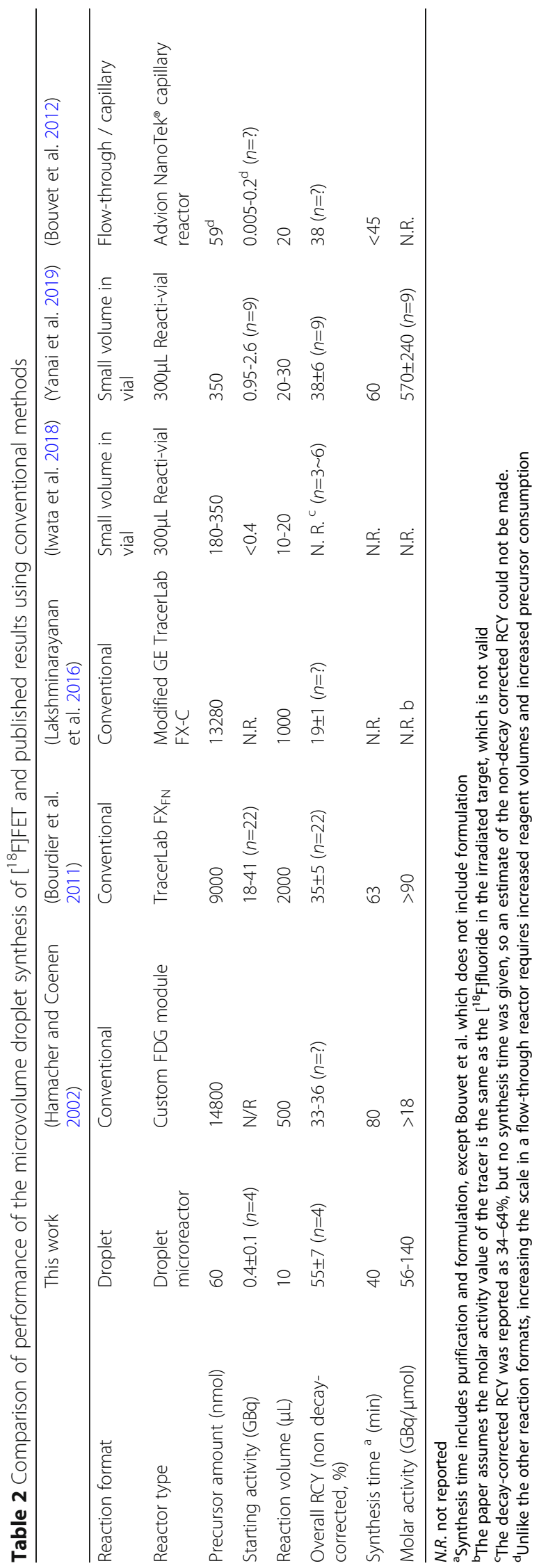


(Heiss et al. 1999; Langen et al. 2003). The results suggest that the $\left[{ }^{18} \mathrm{~F}\right] \mathrm{FET}$ transporters on the lung cancer cell line HCC827 do not become saturated within the range of molar activity values tested. Though the in vitro experiments suggest that the transporters can be "saturated" with sufficient concentration of FET in the media (i.e., 2.5 $\mathrm{mM}$ in the case "spiked" with FET; $0.15 \mu \mathrm{M}$ in the non-spiked condition), the estimated concentration of FET in blood during the in vivo experiments was much lower (i.e. $3.5 \mu \mathrm{M}$ for the lowest molar activity of $0.37 \mathrm{GBq} / \mu \mathrm{mol}$, assuming $2 \mathrm{~mL}$ blood volume).

\title{
Conclusion
}

In this work the synthesis of $\left[{ }^{18} \mathrm{~F}\right] \mathrm{FET}$ was adapted to an automated microdroplet synthesis platform (Wang et al. 2017). The product was obtained in high RCY of up to $55 \pm 7 \%$ ( $n=4$, decay-corrected) after purification, in sufficient quantities to perform a demonstration of a multi-animal dynamic PET imaging study, and could readily be scaled to higher amounts using radionuclide concentration methods (Chao et al. 2018a). Synthesis time was shorter than conventional approaches, precursor consumption was reduced by two orders of magnitude, and the synthesis could be performed with a very small apparatus. The low precursor consumption enabled faster and simpler purification (i.e., analytical HPLC instead of semi-preparative HPLC), and, for tracers with expensive precursors, could help to reduce the synthesis cost. The molar activity was high $(48-119 \mathrm{GBq} / \mu \mathrm{mol}$ at the end of synthesis), even when starting with activities as low as $0.3 \mathrm{GBq}$. Though low molar activity of $\left[{ }^{18} \mathrm{~F}\right] \mathrm{FET}$, (down to $0.37 \mathrm{GBq} / \mu \mathrm{mol}$ ) did not appear to adversely affect imaging of subcutaneous tumors in this study, the ability to produce small batches with high molar activity may be important in other applications of this or other tracers.

\section{Supplementary information}

Supplementary information accompanies this paper at https://doi.org/10.1186/s41181-019-0082-3.

Additional file 1. Supplemental information.

\author{
Abbreviations \\ $\left[{ }^{18} \mathrm{~F}\right] \mathrm{FET}$ : O-(2-[8 F]fluoroethyl)-L-tyrosine; CT: Computed tomography; ESM: Electronic supplementary material; \\ HPLC: High-performance liquid chromatography; NSG: NOD scid gamma; PET: Positron emission tomography; \\ RCY: Radiochemical yield; TLC: Thin-layer chromatography
}

\begin{abstract}
Acknowledgements
We thank Roger Slavik and the staff of the UCLA Biomedical Cyclotron Facility for generously providing $\left[{ }^{18} \mathrm{~F}\right]$ fluoride for these studies, David Nathanson for providing glioblastoma cell cultures and murine models for preliminary studies, and Jason Lee and Dishan Abeydeera for their assistance with performing preclinical imaging. We thank Arion Chatziioannou for letting us use the HiPET prototype scanner for in vivo studies. Microfluidic substrates were produced in the UCLA Integrated NanoSystems Cleanroom (ISNC), and we thank the staff for technical support.
\end{abstract}

Authors' contributions

$\mathrm{KL}, \mathrm{BYC}, \mathrm{JW}, \mathrm{PMC}, \mathrm{RMVD}$ designed experiments and analyzed data. KL performed manual radiochemistry experiments and analyzed data, JW performed set up and operation of the automated radiosynthesizer, and KMF assisted in early development of synthesis protocols. BYC and PMC performed in vitro assays and prepared mouse models. KL and PMC performed imaging analysis. All authors contributed to writing the manuscript, and all authors approved the final version of the manuscript. Biomedical Imaging and Bioengineering (T32 EB002101), and the UCLA Foundation from a donation made by Ralph and Marjorie Crump for the Crump Institute for Molecular Imaging. 
Availability of data and materials

All data generated or analyzed during this study are included in this published article and its supplementary information files. Additional raw measurements are available from the corresponding author on reasonable request.

\section{Ethics approval and consent to participate}

Animal studies were approved by the UCLA Animal Research Committee and were carried out according to the guidelines of the Division of Laboratory Animal Medicine at UCLA.

\section{Consent for publication}

Not applicable.

\section{Competing interests}

Dr. van Dam is a founder of Sofie, Inc. The Regents of the University of California have licensed technology to Sofie related to this work that was invented by Dr. van Dam, and have taken equity in Sofie as part of the licensing transaction.

\section{Author details}

${ }^{1}$ Physics in Biology and Medicine Interdepartmental Graduate Program, University of California, Los Angeles, Los Angeles, CA, USA. ${ }^{2}$ Crump Institute for Molecular Imaging, University of California, Los Angeles, Los Angeles, CA, USA. ${ }^{3}$ Department of Molecular \& Medical Pharmacology, David Geffen School of Medicine, University of California, Los Angeles, Los Angeles, CA, USA. ${ }^{4}$ Department of Bioengineering, University of California, Los Angeles, Los Angeles, CA, USA.

Received: 8 October 2019 Accepted: 21 November 2019

Published online: 31 December 2019

\section{References}

Aboagye EO, Price PM, Jones T. In vivo pharmacokinetics and pharmacodynamics in drug development using positronemission tomography. Drug Discov Today. 2001;6(6):293-302.

Ambrosini V, Quarta C, Nanni C, Pettinato C, Franchi R, Grassetto G, et al. Small animal PET in oncology: the road from bench to bedside. Cancer Biother Radiopharm. 2009;24(2):277-85.

Bourdier T, Greguric I, Roselt P, Jackson T, Faragalla J, Katsifis A. Fully automated one-pot radiosynthesis of O-(2[18F]fluoroethyl)-I-tyrosine on the TracerLab FXFN module. Nucl Med Biol. 2011;38(5):645-51.

Bouvet V, Wuest M, Tam P-H, Wang M, Wuest F. Microfluidic technology: an economical and versatile approach for the synthesis of O-(2-[18F]fluoroethyl)-L-tyrosine ([18F]FET). Bioorg Med Chem Lett. 2012;22(6):2291-5.

Chao PH, Lazari M, Hanet S, Narayanam MK, Murphy JM, van Dam RM. Automated concentration of [18F]fluoride into microliter volumes. Appl Radiat Isot. 2018a;141:138-48.

Chao PH, Wang J, Van Dam RM. A fully automated radiosynthesis platform for scalable production and purification of PET tracers. In: Proc. 22nd Int. Conf. Miniaturized Syst. Chem. Life Sci. Kaohsiung, Taiwan: Royal Society of Chemistry; 2018b. p. 1155-8.

Ciernik F, Dizendorf E, Baumert B, Reiner B, Burger C, Davis B, et al. Radiation treatment planning with an integrated positron emission and computer tomography (PET/CT): a feasibility study. Int J Radiat Oncol Biol Phys. 2003;57(3):853-63.

Dooraghi AA, Keng PY, Chen S, Javed MR, Kim C-J, Chatziioannou AF, et al. Optimization of microfluidic PET tracer synthesis with Cerenkov imaging. Analyst. 2013;138(19):5654-64.

Elizarov AM. Microreactors for radiopharmaceutical synthesis. Lab Chip. 2009;9(10):1326-33.

Elizarov AM, van Dam RM, Shin YS, Kolb HC, Padgett HC, Stout D, et al. Design and optimization of coin-shaped microreactor chips for PET radiopharmaceutical synthesis. J Nucl Med. 2010 Feb 1;51(2):282-7.

Gu Z, Taschereau R, Prout DL, Vu N, Chatziioannou A. Performance evaluation of HiPET, a high sensitivity and high spatial resolution DOI PET tomograph. Atlanta: IEEE NPSS; 2017. Available from: https://www.eventclass.org/contxt_ieee2017/ online-program/session?s=M-08\#3593

Hamacher K, Coenen HH. Efficient routine production of the 18F-labelled amino acid O-(2-[18F]fluoroethyl)-I-tyrosine. Appl Radiat Isot. 2002;57(6):853-6.

Heiss P, Mayer S, Herz M, Wester H-J, Schwaiger M, Senekowitsch-Schmidtke R. Investigation of transport mechanism and uptake kinetics of O-(2-[18F]Fluoroethyl)-L-tyrosine in vitro and in vivo. J Nucl Med. 1999;40(8):1367-73.

Iwata R, Pascali C, Terasaki K, Ishikawa Y, Furumoto S, Yanai K. Practical microscale one-pot radiosynthesis of 18F-labeled probes. J Label Compd Radiopharm. 2018;61(7):540-9.

Keng PY, van Dam RM. Digital microfluidics: a new paradigm for radiochemistry. Mol Imaging. 2015;14:579-94.

Keng PY, Sergeev M, van Dam RM. Advantages of radiochemistry in microliter volumes. In: Kuge Y, Shiga T, Tamaki N, editors. Perspect. Nucl. Med. Mol. Diagn. Integr. Ther. [Internet]. Tokyo: Springer Japan; 2016. p. 93-111. [cited 2016 Apr 11]. Available from: http://link.springer.com/chapter/10.1007/978-4-431-55894-1_7.

Kitson S, Cuccurullo V, Ciarmiello A, Salvo D, Mansi L. Clinical applications of positron emission tomography (PET) imaging in medicine: oncology. Brain Dis Cardiol Curr Radiopharm. 2009;2(4):224-53.

Kuik W-J, Kema IP, Brouwers AH, Zijlma R, Neumann KD, Dierckx RAJO, et al. In vivo biodistribution of no-carrier-added 6-18FFluoro-3,4-Dihydroxy-L-phenylalanine (18F-DOPA), produced by a new Nucleophilic substitution approach, compared with carrier-added 18F-DOPA, prepared by conventional electrophilic substitution. J Nucl Med. 2015;56(1):106-12.

Lakshminarayanan N, Kumar A, Roy S, Pawar Y, Chaudhari PR, Rajan MGR. Fully automated synthesis of O-(2'[18F]fluoroethyl)-I-tyrosine ([18F]FET) using solid phase extraction (SPE) purification with neutral alumina. J Radioanal Nucl Chem. 2016;11:1-9.

Langen K-J, Jarosch M, Mühlensiepen H, Hamacher K, Bröer S, Jansen P, et al. Comparison of fluorotyrosines and methionine uptake in F98 rat gliomas. Nucl Med Biol. 2003 May 1;30(5):501-8. 
Langen K-J, Hamacher K, Weckesser M, Floeth F, Stoffels G, Bauer D, et al. O-(2-[18F]fluoroethyl)-I-tyrosine: uptake mechanisms and clinical applications. Nucl Med Biol. 2006 Apr 1;33(3):287-94.

Langen K-J, Stoffels G, Filss C, Heinzel A, Stegmayr C, Lohmann P, et al. Imaging of amino acid transport in brain tumours: positron emission tomography with O-(2-[18F]fluoroethyl)-L-tyrosine (FET). Methods. 2017;130:124-34.

Lebedev A, Miraghaie R, Kotta K, Ball CE, Zhang J, Buchsbaum MS, et al. Batch-reactor microfluidic device: first human use of a microfluidically produced PET radiotracer. Lab Chip. 2012;13(1):136-45.

Miller PW, de Mello AJ, Gee AD. Application of Microfluidics to the Ultra-Rapid Preparation of Fluorine-18 Labelled Compounds. Curr Radiopharm. 2010;3:254-62.

Pascali G, Matesic L. How far are we from dose on demand of short-lived radiopharmaceuticals? In: Kuge Y, Shiga T, Tamaki N, editors. Perspect. Nucl. Med. Mol. Diagn. Integr. Ther. [Internet]. Tokyo: Springer Japan; 2016. p. 79-92. [cited 2016 Sep 26]. Available from: http://link.springer.com/chapter/10.1007/978-4-431-55894-1_6.

Pascali G, Watts P, Salvadori P. Microfluidics in radiopharmaceutical chemistry. Nucl Med Biol. 2013 Aug;40(6):776-87.

Phelps ME. PET: the merging of biology and imaging into molecular imaging. J Nucl Med. 2000;41(4):661-81.

Rensch C, Jackson A, Lindner S, Salvamoser R, Samper V, Riese S, et al. Microfluidics: a groundbreaking technology for PET tracer production? Molecules. 2013 Jul 5;18(7):7930-56.

Sergeev M, Lazari M, Morgia F, Collins J, Javed MR, Sergeeva O, et al. Performing radiosynthesis in microvolumes to maximize molar activity of tracers for positron emission tomography. Commun Chem. 2018a;1(1):10

Sergeev M, Lazari M, Morgia F, Collins J, Javed MR, Sergeeva O, et al. Performing radiosynthesis in microvolumes to maximize molar activity of tracers for positron emission tomography. Commun Chem. 2018b;1(1):10.

Taschereau R, Vu NT, Chatziioannou AF. Calibration and data standardization of a prototype bench-top preclinical CT. In: 2014 IEEE Nucl. Sci. Symp. Med. Imaging Conf. NSSMIC; 2014. p. 1-2.

Wang J, Chao PH, Hanet S, van Dam RM. Performing multi-step chemical reactions in microliter-sized droplets by leveraging a simple passive transport mechanism. Lab Chip. 2017;17(24):4342-55.

Wester HJ, Herz M, Weber W, Heiss P, Senekowitsch-Schmidtke R, Schwaiger M, et al. Synthesis and Radiopharmacology of O-(2-[18F]fluoroethyl)-L-tyrosine for tumor imaging. J Nucl Med. 1999;40(1):205-12.

Yanai A, Harada R, Iwata R, Yoshikawa T, Ishikawa Y, Furumoto S, et al. Site-specific labeling of F-18 proteins using a supplemented cell-free protein synthesis system and O-2-[18F]Fluoroethyl-L-tyrosine: [18F]FET-HER2 Affibody molecule. Mol Imaging Biol. 2019;21(3):529-37.

\section{Publisher's Note}

Springer Nature remains neutral with regard to jurisdictional claims in published maps and institutional affiliations.

\section{Submit your manuscript to a SpringerOpen ${ }^{\circ}$ journal and benefit from:}

- Convenient online submission

- Rigorous peer review

- Open access: articles freely available online

High visibility within the field

Retaining the copyright to your article

Submit your next manuscript at $\boldsymbol{\nabla}$ springeropen.com 